\title{
In Vitro Comparative Quality Evaluation of Non-Expired and 10 Years-Expired Lamotrigine Immediate-Release Tablet Formulations - Pilot Study
}

\author{
Gordana Švonja Parezanović ${ }^{*}$, Mladena Lalić-Popović ${ }^{1}$, Svetlana Goločorbin-Kon ${ }^{1}$, Nemanja \\ Todorović ${ }^{1}$, Nebojša Pavlović1 ${ }^{\text {, and Momir Mikov }}{ }^{2}$ \\ ${ }^{1}$ Faculty of Medicine, Department of Pharmacy, University of Novi Sad, Serbia. \\ ${ }^{2}$ Faculty of Medicine, Department of Pharmacology, Toxicology and Clinical Pharmacology, University Novi Sad, Serbia.
}

\section{ABSTRACT}

e-mail: gordana.svonja-parezanovic@mf.uns.ac.rs

This study aimed to compare the different physical parameters and dissolution profiles of 10 years-expired with nonexpired lamotrigine (LTG) immediate-release tablet formulations. Dissolution tests were conducted using a validated high-performance liquid chromatography method. Dissolution characteristics of the tablet formulations were evaluated at three points spanning the physiological $\mathrm{pH}$ range $(\mathrm{pH} 1.2, \mathrm{pH} 4.5, \mathrm{pH}$ 6.8). Physical characteristics of LTG tablets were determined according to the European Pharmacopoeia. The dissolution profile comparison was carried out using model-independent (statistical) and model-dependent (kinetic) methods to provide detailed information about dissolution data. The t-test was applied to investigate differences between expired and non-expired tablets, and the differences were considered statistically significant if $p \leq 0.05$. The results demonstrated no statistical differences in physical characteristics between expired and non-expired tablets; however, there were differences in the dissolution profiles, which could cause different pharmacokinetic profiles. The results of this investigation showed that the drug content did not change significantly. Therefore, the lower dissolution rate for expired LTG tablets is due to an interaction of LTG with one or more excipients in the tablet formulation during aging.

KEYWORDS: Lamotrigine, expiration, dissolution, quality, interaction

\section{INTRODUCTION}

D rug stability is a quality control attribute for pharmaceutical dosage forms that describes the ability of the drug to maintain the physical, chemical, therapeutic, and microbial properties during the time of storage (1). The expiration date specifies the time period during which the product is known to remain stable, which means the manufacturer guarantees the drug retains its quality and purity when it is stored according to its labeled storage conditions (2). The United States Food and Drug Administration (US FDA) requires stability data from pharmaceutical companies submitting new drug applications to establish shelf-life specifications (1). Stability studies are designed to provide confidence that the product will meet the applicable requirements for quality, safety, and efficacy throughout the shelf-life (1). The FDA verifies that an applicant's proposed expiration date is supported by appropriate studies conducted by the applicant.

Expired pharmaceutical products have not necessarily lost potency, because the expiration date is only an assurance that the labeled potency will last at least until that time. Most pharmaceutical products continue to be effective and safe for a time after the expiration date (3-6).

Many studies have shown that most active pharmaceutical ingredients (APIs) retain their pharmacological potency far beyond the expiration date. A study conducted by the US FDA evaluated over 100 pharmaceutical dosage forms and showed that about $90 \%$ of them were safe and effective as long as 15 years past their expiration dates (5). Another other study had similar results, showing that many drugs stored under reasonable conditions retain $90 \%$ of their potency for at least 5 years after the expiration date on the label and sometimes much longer (3). Also, it is determined that four products, namely captopril tablets, flucloxacillin capsules, cefoxitin injection, and theophylline tablets, when stored under ambient temperatures maintained at least $98 \%$ of label claim for drug content for 18-170 months past the labeled expiration dates (6).

Consequently, the FDA and the United States

* Corresponding author. 
Pharmacopeia (USP) in cooperation with pharmaceutical industry recommended to determine the benefits and risks associated with lengthening expiration dates and to subsequently conduct longer stability testing. The initial expiration date is based on the amount of real-time stability data for the pharmaceutical product available at the time of approval of the FDA. This initial date can be extended contingent upon the receipt of acceptable supporting data from the manufacturer based on accelerated stability studies. Generally, the marketed pharmaceutical products have a labeled shelf life of 1260 months.

Regarding the expired medications, most studies have focused on degradation of active pharmaceuticals, so the knowledge of the pharmaceutical performance is limited. There are not many studies on the pharmaceutical quality of expired products; only a few studies have addressed the long-term stability of pharmaceutical dosage forms.

Lamotrigine (LTG) is indicated in the treatment of different types of epilepsy and in bipolar disorder, effective as an add-on therapy in the management of simple and complex partial seizures and secondarily generalized tonic-clonic seizures resistant to multipledrug therapy (7).

LTG is in class II according to the Biopharmaceutical Classification System (BCS), a lipophilic, weak base poorly soluble in water with a log octanol/water partition coefficient of 1.19 at $\mathrm{pH} 7.6$ (8). However, its poor aqueous solubility $(0.17 \mathrm{mg} / \mathrm{mL})$ confines its absorption and dissolution rate and hence delay onset of action (9). In general, dissolution of BCS class II drugs is dependent on a wide variety of physiological factors like $\mathrm{pH}$, ionic strength, and buffer capacity, which are three major characteristics of the gastrointestinal (GI) fluids that can affect the rate of drug release (9). Thus, drug dissolution may be the rate-limiting step for drug absorption, and an in vivo-in vitro correlation may be expected. The FDA guidelines recommend analysis of dissolution profiles of BCS class II drugs in multiple dissolution media with a $\mathrm{pH}$ range 1.2 to 6.8 (1). Factors that influence drug dissolution from tablet forms, albeit physicochemical properties of the active ingredients, are the nature and quantity of excipients and their interactions, compression pressure, speed of compressions, condition of storage, and age of the tablet (10-12).

The ability of a drug to have an extended shelf life would be dependent upon the actual drug ingredients, presence of excipients, and storage conditions. For drugs of BCS class 2, a two-point dissolution specification, one at 15 minutes to include a dissolution range and the other at a later point $(30,45$, or 60 minutes) to ensure $85 \%$ dissolution, is recommended to characterize the quality of the product. The $\mathrm{pH}$ of the $\mathrm{Gl}$ tract influences the dissolution rate of a drug (13). Weakly basic BCS class II drugs such as LTG tend to have a slower dissolution rate at the higher $\mathrm{pH}$ of $\mathrm{Gl}$ fluid during transition from the stomach to the intestine (14-16).

The aim of this pilot study is to determine the quality of LTG immediate-release tablets 10 years after the expiration date with those that are not expired. The tablets were subjected to various post-production tests such as determination of hardness, weight, friability, disintegration time, content determination, and dissolution testing.

\section{MATERIALS AND METHODS}

Two tablets containing LTG were tested: tablet A (reference, non-expired tablets), which was commercially available and randomly chosen, and tablet $B$ (test, expired tablets), which was produced by the same manufacturer but 10 years past the expiration date and returned to the local pharmacy by the patient. The labeled amount of drug substance was $100 \mathrm{mg}$ per tablet.

\section{Chemicals and Reagents}

LTG (99\% purity) standard was provided by Alakloid (Skopje, Macedonia) and chloramphenicol from Galenika (Belgrade, Serbia). Acetonitrile and methanol were highperformance liquid chromatography (HPLC) grade and obtained from CarloErba (Milano, Italy). Orthophosphoric acid 85\% (Lanchema a.s., Czech Republic) and potassium dihydrogen phosphate $(\mathrm{POCH}$, Poland) were analytical reagent grade.

Excipients added in formulation of these tablets were lactose monohydrate, microcrystalline cellulose, povidone $\mathrm{K} 30$, sodium starch glycolate (type A), iron oxide yellow (E172), and magnesium stearate.

\section{High-Performance Liquid Chromatography (HPLC) analyses}

The HPLC system (Agilent Technology Series 1100, Wilmington, DE, USA) used was equipped with degasser, binary pump, automatic injector, and diode array detector (DAD) detector G1315B, with software for data processing (Agilent ChemStation). Chromatographic separation was performed on a C18 guard column $(4.6 \times 12.5 \mathrm{~mm}, 5 \mu \mathrm{m}$, Zorbax) and C18 analytical column $(4.6 \times 250 \mathrm{~mm}, 5 \mu \mathrm{m}$, Zorbax). The assay of active substance was performed according to a previously published HPLC method for determination of LTG in dissolution medium and tablet 
formulation (17). The mobile phase was a mixture of 0.05 $\mathrm{M}$ ammonium dihydrogen phosphate (adjusted $\mathrm{pH}=2.68$ with $40 \%$ ortho-phosphoric acid) and acetonitrile (68:32, $\mathrm{v} / \mathrm{v})$. All separations were performed isocratically at a flow rate of $1.2 \mathrm{~mL} / \mathrm{min}$, and column temperature was maintained at room temperature $\left(25^{\circ} \mathrm{C}\right)$. For quantitative determination, the peak areas were determined using a calibration curve and external standard (chloramphenicol) with DAD detector set at wavelength $265 \mathrm{~nm}$.

\section{Tablet Quality Characteristics}

The quality of LTG tablets was determined according to the 9th European Pharmacopoeia (EP) (18). The content uniformity test was performed with 10 tablets from each series that were weighed individually and each of the 10 tablets finely powdered and dissolved in the mobile phase. A standard solution was prepared by dissolving pure LTG in mobile phase. The concentrations of prepared solutions were determined by HPLC method mentioned above. According to the $E P$, content uniformity should be between $85 \%$ and $115 \%$ of the average content value. Also, 20 tablets were randomly selected from each series and weighed individually and together with an electronic balance for calculation of weight variation. The average weight was noted, and relative standard deviation (RSD) was calculated. The limit of RSD for weight variation in case of tablets of $100 \mathrm{mg}$ mass is $\pm 7.5 \%$ (18).

The disintegration test was carried out using the disintegration tester for six tablets. The basket was immersed in a bath of water at $37^{\circ} \mathrm{C}$ in a 1-L beaker until the tablet was disintegrated, according to the EP. The $E P$ does not have a requirement for the hardness of the tablet; however, some researchers state that the crushing force of immediate-release tablets should be between 50-100 N (or 120 N) (19). The hardness test was applied with a hardness tester (Erweka, Heusenstamm, Germany) on 10 tablets for each examined series.

Friability test was performed with 10 tablets from each series, which were weighed and put into the friabilator (Erweka). Tablets were rotated at $25 \mathrm{rpm}$ for 5 minutes six times, and the friability percentage was calculated for each tablet. A maximum weight loss (obtained from a single test or from the mean of three tests) of not more than $1.0 \%$ is considered acceptable.

\section{Dissolution Testing}

Dissolution profiles were evaluated according to the United States Pharmacopoeia (USP paddle method (apparatus 2) (20). The dissolution test was performed on a tablet dissolution tester (CD800, Erweka). The dissolution profile of LTG tablets was evaluated in $\mathrm{pH} 1.2$ hydrochloric acid ( $\mathrm{HCl}$ ) buffer, $\mathrm{pH} 4.5$ acetate buffer, and pH 6.8 phosphate buffer.

\section{Mathematical Models and Statistical Analyses}

The t-test was applied to investigate differences between expired and non-expired tablets and the differences were considered statistical if $p \leq 0.05$. The dissolution profile comparison was carried out using model independent statistical, and model dependent methods to evaluate the dissolution data. The model-independent method used the difference $\left(f_{1}\right)$ and similarity factors $\left(f_{2}\right)$, recommended by the FDA Guidance for Industry to compare dissolution profiles of both LTG tablets (2). Generally, $f_{1}$ values up to $15(0-15)$ and $f_{2}$ values greater than 50 (50-100) ensure sameness or equivalence of the two curves of compared products.

The model-dependent method included several kinetic models in which the dissolved amount of drug is a function of time, are used to describe drug dissolution from solid dosage forms (21). The kinetics of the dissolution process were studied through the analysis dissolution data using five kinetic models: first-order, Hixson-Crowell's cube root law, Higuchi's square root equation, Weibull's distribution, and the logistic model (22-26). The best fitting equation uses coefficient of determination $\left(R^{2}\right)$, Akaike information criterion (AIC), and model selection criterion (MSC) (22). The drug release kinetics and mechanism were computed using DDSolver (menu-driven add-in program for Microsoft Excel written in Visual Basic for Applications) (27).

\section{RESULTS AND DISCUSSION}

The results of our study are presented in Table 1, including analysis of drug content, physical characteristics (weight, hardness, friability, disintegration time), and estimation of dissolution profiles of non-expired $(A)$ and 10 yearsexpired (B) LTG tablet formulations. The average weight deviation of 20 tablets taken from each formulation was less than $\pm 3 \%$, and all the formulations met the requirement (Table 1 ).

Table 1. Physical Characteristics of Non-Expired (A) and Expired(B) Lamotrigine Tablets

\begin{tabular}{|c|c|c|c|}
\hline & Tablet A & Tablet B & $p$ value \\
\hline Content uniformity (\%) & $100.02 \pm 7.65$ & $98.72 \pm 5.81$ & $>0.05$ \\
\hline Weight (g) & $0.3989 \pm 0.0027$ & $0.3995 \pm 0.0018$ & $>0.05$ \\
\hline $\begin{array}{c}\text { Disintegration time } \\
\text { (min) }\end{array}$ & $2.21 \pm 0.04$ & $1.76 \pm 0.10$ & $>0.05$ \\
\hline Hardness (kg/mm²) & $8.4 \pm 0.5$ & $7.6 \pm 0.9$ & $>0.05$ \\
\hline Friability (\%) & 0.572 & 0.574 & $>0.05$ \\
\hline
\end{tabular}

Data are expressed as mean $\pm S D$. 
The tested LTG tablets showed no differences in hardness test results. Since tablet hardness is not a perfect index to evaluate the strength of the tablets, friability was also used to test the hardness of tablets. For the both tablet series, friability percentage was less than $1 \%$, which is in the acceptable range (18). Content uniformity test results showed that both LTG tablets fit the criteria of EP and were in accordance with the claimed value $(100 \mathrm{mg})$ by manufacturer. Both LTG tablets contain at least $92.5 \%$ and not more than $107.5 \%$ of the labeled amount of the active drug.

All analyzed tablets disintegrated in 15 minutes, according to $9^{\text {th }} E P$ test of uncoated tablets (18). A paired $t$ test showed no statistically significant difference of the physical characteristics of expired and non-expired tablet series (Table 1).

Though the physical characteristics of tablets and content could remain the same, aging of tablets could lead to alteration in dissolution profiles and kinetics of release. Thus, the absence of dissolution changes would provide assurance that the bioavailability for therapeutic efficiency remained unchanged 10 years after the expiration date. Significant changes in vitro release could affect therapeutic efficiency and, in the case of LTG, epileptic seizure relapse could occur.

Dissolution profiles of the two examined tablets are showed in Figures 1-3. As expected, the fastest in vitro drug release rate was obtained in more acidic $\mathrm{pH}$, which is a reflection of the highest solubility of the drug in $\mathrm{pH} 1.2$ $\mathrm{HCl}$ buffer (Fig. 1). Therefore, in the dissolution medium $\mathrm{pH} 1.2$, tablet A showed almost complete release after 15 $\min (97 \%)$, contrarily the tablets with expired date showed much slower drug release, only $45.07 \%$ at $15 \mathrm{~min}$. The reason for differences in dissolution profile between the investigated tablets could be interaction with excipients. Physical interactions between excipients are very difficult to detect because there are no chemical changes involved (28). One example of a physical interaction is interactive mixing. This is specific for smaller particles of pharmaceuticals that interact with the surface of larger particles of ingredients in the drug formulation, usually excipients (29).

As shown in Figure 2, the rate of LTG tablet B release in acetate buffer ( $\mathrm{pH} 4.5)$ was after 35 minutes faster than tablet A. For tablet B, $47.43 \%$ of drug released within 5 min in acetate buffer, and $65.43 \%$ (incompletely dissolved) within the first $15 \mathrm{~min}$. Expired tablets of LTG (B) have faster release in $\mathrm{pH} 4.5$ than $\mathrm{pH} 1.2$.

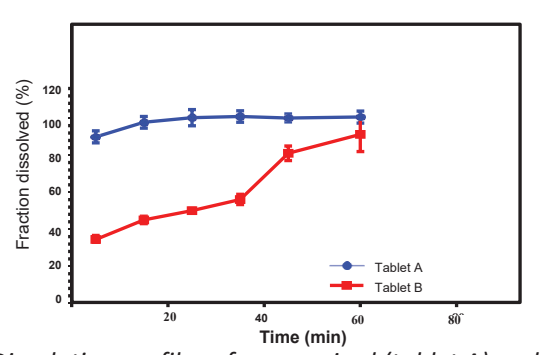

Figure 1. Dissolution profiles of non-expired (tablet $A$ ) and 10 years-expired (tablet $B$ ) lamotrigine tablets in $\mathrm{pH} 1.2$ dissolution medium.

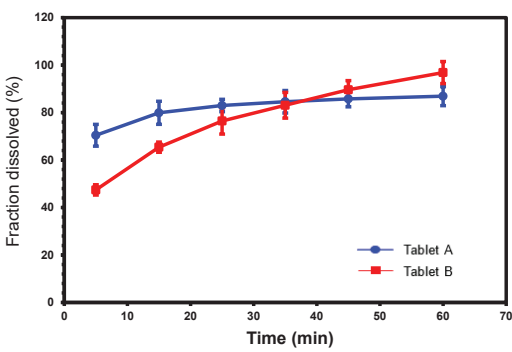

Figure 2. Dissolution profiles of non-expired (tablet A) and 10 years-expired (tablet $B$ ) lamotrigine tablets in $\mathrm{pH} 4.5$ dissolution medium.

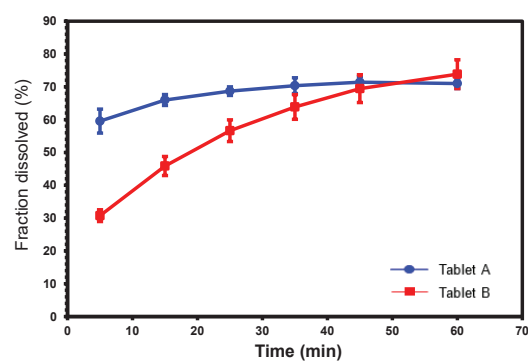

Figure 3. Dissolution profiles of non-expired (tablet A) and 10 years-expired (tablet $B$ ) lamotrigine tablets in $\mathrm{pH} 6.8$ dissolution medium.

Magnesium stearate is a common lubricant used for solid pharmaceutical formulations (30). Because of its hydrophobicity, during aging magnesium stearate can also delay tablet dissolution (30-31). The presence of magnesium stearate in a formulation with aging can increase the micro-environmental $\mathrm{pH}$ of the formulation, creating an alkaline condition and cause delay of tablet dissolution. Thus, by using this phenomenon when magnesium stearate was added, the tablet tended to float on the surface of $\mathrm{pH} 1.2$ or $\mathrm{pH} 4.5$ dissolution media; however, it dispersed in the $\mathrm{pH} 6.8$ buffer. The slowest rate of release was obtained in $\mathrm{pH} 6.8$ phosphate buffer, which is a consequence of the low solubility in alkaline medium (Fig. 3).

Dissolution from the tablet dosage form involves liberation of the drug from the formulation matrix (disintegration) followed by the dissolution of the drug (solubilization of the drug particles) in the liquid medium. Because the release of LTG from tablets with expired date $(B)$ is slower from the start compared to unexpired tablets $(A)$, the characteristics of the disintegrator could be connected with this delay of release. The ionic nature of the drugs 
and superdisintegrants and their potential interactions have been reported to affect dissolution stability (32). Consequently, accelerated release of expired tablets after 35 min of dissolution has been noticed and could be the moment when disintegration of tablets is finished. In the first step of dissolution, the cohesive properties of the formulated drug play a key role. Also, it has been reported that aging decreases the dissolution efficiency of super disintegrants in wet granulated tablets, which confirms the previous theory $(33,34)$.

In Table 2, results of dissolution profile comparison are shown. The similarity factor $\left(f_{2}\right)$ is more sensitive in finding dissimilarity between dissolution curves than the difference factor $\left(f_{1}\right)$, and the values of fit factors are dependent on the number of sampling time points chosen $(35,36)$. According to the FDA guidelines, $f_{1}$ values up to 15 and $f_{2}$ values greater than 50 should ensure equivalence of the dissolution curves, indicating an average difference of no more than $10 \%$ at the sample time points. Based on these guidelines, the tablets showed dissolution curve equivalence only in the $\mathrm{pH} 4.5$ acetate buffer medium.

Table 2. Dissolution Data for Calculation of Similarity $\left(f_{2}\right)$ and Difference $\left(f_{1}\right)$ Factors for Expired $(B)$ verus Non-Expired $(A)$ Lamotrigine Tablets

\begin{tabular}{|c|c|c|c|}
\hline & \multicolumn{3}{|c|}{ Dissolution Medium } \\
\hline Variable & $\mathrm{pH} \mathrm{1.2}$ & $\mathrm{pH} \mathrm{4.5}$ & $\mathrm{pH} \mathrm{6.8}$ \\
\hline$f_{1}$ & 39.27 & 12.08 & 17.76 \\
\hline$f_{2}$ & 18.59 & 45.55 & 40.50 \\
\hline $\mathbf{5} \mathbf{~ m i n}$ & $p<0.0001$ & $p<0.0001$ & $p<0.0001$ \\
\hline $\mathbf{1 0} \mathbf{~ m i n}$ & $p<0.0001$ & $p<0.0001$ & $p<0.0001$ \\
\hline $\mathbf{1 5} \mathbf{m i n}$ & $p<0.0001$ & $p<0.05$ & $p<0.0001$ \\
\hline $\mathbf{3 0} \mathbf{m i n}$ & $p<0.0001$ & $p<0.05$ & $p<0.0001$ \\
\hline $\mathbf{4 5} \mathbf{m i n}$ & $p<0.0001$ & $p<0.05$ & $p<0.05$ \\
\hline $\mathbf{6 0} \mathbf{~ m i n}$ & $p<0.05$ & $p<0.05$ & $p<0.05$ \\
\hline
\end{tabular}

Further, it is shown in Table 2 that there are statistically significant differences in dissolution profiles $(p<$ 0.0001 and $<0.05$ ) between tablets $A$ and $B$ in multiple dissolution media ( $\mathrm{pH}$ 1.2, $\mathrm{pH} 4.5, \mathrm{pH}$ 6.8). To understand the mechanism of drug release, data were fitted to different kinetic models (Table 3). This method presents an acceptable model-based approach in finding the true relationship between percent dissolved and time variables of the two dissolution profiles. Selection of the best and most accurate models should be based on appropriate criteria such as $R^{2}, \mathrm{AIC}$, and MSC. The best-fit model may be considered with lesser AIC and higher MSC and $R^{2}$ adjusted values. According to previously established criteria for choosing the best kinetic model, Table 3 clearly depicted that tablet $A$ in the $\mathrm{pH} 1.2$ dissolution medium followed Weibull's model. The correlation coefficient values of the Weibull's model are found to be slightly higher when compared to the first order, logistic, and Korsmeyer-Peppas release models. In the same medium, tablets with expired date (B) followed the Korsmeyer-Peppas model. This model is generally used to analyze the release of pharmaceutical exponents, which indicate the drug release from polymeric dosage forms when the release mechanism is not well known or when more than one type of release phenomena is involved. Also, tablet B had slightly lower correlation coefficient values of the Higuchi's model $\left(R^{2}=0.9477\right.$, AIC $=41.5736, \mathrm{MSC}=2.0131$ ), which is significantly different than the correlation coefficient values for tablet $\mathrm{B}\left(R^{2}=\right.$ 0.3898, $\mathrm{AIC}=61.7639, \mathrm{MSC}=-1.3898$ ).

In the $\mathrm{pH} 4.5$ dissolution medium, the Weibull model was the predominant release mechanism for tablet $A$, but tablet B predominantly followed the Korsmeyer-Peppas model. Release kinetics of tablet $\mathrm{A}$ in $\mathrm{pH} 6.8$ dissolution medium best fit the Korsmeyer-Peppas model, which was the same as tablet B. Consequently, kinetic modeling of the dissolution profiles indicated different models of release for expired and non-expired LTG tablets of the same brand.

\section{CONCLUSIONS}

This pilot study found no differences in physical characteristics between expired and non-expired tablets of LTG; however, there were differences in the dissolution profiles. Expired LTG tablets had lower release than non-expired LTG tablets; hence the expired tablets are expected to have lower effectiveness. The dissolution profiles clearly showed that $\mathrm{pH}$ plays an important role in the dissolution of aging tablets. Similar content uniformity indicates that no degradation of the active ingredient occurred 10 years after expiration date. The results of this investigation showed that the drug content did not change significantly. Therefore, the lower dissolution rate for expired LTG tablets is due to an interaction of LTG with one or more excipients in the tablet formulation during aging. Significant changes in the in vitro release profiles of a drug product during storage may alter its bioavailability. Further studies are essential to verify the clinical efficiency of expired LTG immediate-release tablet formulations stored under unsuitable conditions. 


\begin{tabular}{|c|c|c|c|c|c|c|c|}
\hline \multirow[b]{2}{*}{ Kinetic model } & \multirow[b]{2}{*}{ Parameters } & \multicolumn{2}{|c|}{ pH 1.2} & \multicolumn{2}{|c|}{$\mathrm{pH} 4.5$} & \multicolumn{2}{|c|}{ pH 6.8} \\
\hline & & Tablet A & Tablet B & Tablet A & Tablet B & Tablet A0 & Tablet B \\
\hline \multirow{4}{*}{ First Order } & $R^{2}$ & 0.9993 & 0.8830 & 0.8046 & 0.9294 & 0.3488 & 0.8763 \\
\hline & k & 0.457 & 0.033 & 0.194 & 0.07 & 0.049 & 0.031 \\
\hline & AIC & 14.2753 & 47.2158 & 51.3665 & 45.0235 & 57.1357 & 45.5301 \\
\hline & MSC & 5.1089 & 1.2071 & -0.4281 & 1.3148 & -1.6619 & 0.9299 \\
\hline \multirow{4}{*}{ Hixon Crowel } & $R^{2}$ & 0.5413 & 0.7017 & 0.5782 & 0.8744 & 0.1482 & 0.8076 \\
\hline & k & 0.026 & 0.009 & 0.023 & 0.018 & 0.012 & 0.008 \\
\hline & AIC & 59.7633 & 41.4565 & 56.7531 & 49.0506 & 59.0154 & 48.6222 \\
\hline & MSC & -1.3898 & 0.8762 & -1.1976 & 0.7395 & -1.4262 & 0.4882 \\
\hline \multirow{4}{*}{ Higuchi } & $R^{2}$ & 0.3898 & 0.9477 & 0.4735 & 0.9123 & 0.4856 & 0.9613 \\
\hline & k & 19.911 & 11.266 & 14.15 & 13.972 & 11.770 & 10.515 \\
\hline & AIC & 61.7639 & 41.5736 & 0.3034 & 46.5411 & 55.4856 & 37.3929 \\
\hline & MSC & -1.6752 & 2.0131 & 147.3 & 1.0980 & 41 & 2.0924 \\
\hline \multirow{4}{*}{ Weibull } & $R^{2}$ & 0.9997 & 0.9335 & 1.0000 & 0.9939 & 0.9685 & 0.9990 \\
\hline & k & 1.958 & 3.562 & 0.152 & 0.585 & 0.135 & 0.539 \\
\hline & AIC & 12.0149 & 36.4478 & -19.7698 & 31.9003 & 11.0012 & 15.4534 \\
\hline & MSC & 5.4318 & 1.7110 & 9.7342 & 3.1895 & 2.7908 & 5.2266 \\
\hline \multirow{4}{*}{ Logistic } & $R^{2}$ & 0.9996 & 0.8907 & 0.9999 & 0.9861 & 0.9746 & 0.9966 \\
\hline & k & 4.526 & 2.135 & 0.972 & 2.240 & 0.513 & 1.749 \\
\hline & AIC & 12.2815 & 48.7384 & 1.6122 & 35.6611 & 9.7165 & 22.2765 \\
\hline & MSC & 5.3937 & 0.9896 & 6.6796 & 2.6523 & 3.0049 & 4.2518 \\
\hline \multirow{4}{*}{$\begin{array}{c}\text { Korsmeyer- } \\
\text { Peppas }\end{array}$} & $R^{2}$ & 0.9984 & 0.9481 & 0.9961 & 0.9999 & 0.9989 & 0.9980 \\
\hline & $k$ & 4.526 & 11.996 & 0.972 & 30.195 & 53.617 & 17.944 \\
\hline & AIC & 22.2205 & 43.5307 & 1.9533 & 3.4336 & 14.2339 & 18.6361 \\
\hline & MSC & 3.9739 & 1.7335 & 4.8870 & 7.2562 & 4.4669 & 4.7719 \\
\hline
\end{tabular}

$R^{2}$, coefficient of determination; AIC, Akaike's information criterion; MSC, model selection criterion.

\section{ACKNOWLEDGMENT}

This work was supported by Ministry of Science and Technology development of Serbia (no 41012).

\section{CONFLICT OF INTEREST}

The authors disclosed no conflicts of interest related to this article.

\section{REFERENCES}

1. International Conference on Harmonisation of Technical Requirements for Registration of Pharmaceuticals for Human Use. Stability Testing of New Drug Substances and Products, Q1A(R2); ICH Harmonized Tripartite Guideline: Geneva, Switzerland, 2003

2. SUPAC-MR: Modified Release Solid Oral Dosage Forms; ScaleUp and Post-Approval Changes: Chemistry, Manufacturing and Controls. Vitro Dissolution Testing, and In Vivo Bioequivalence Documentation; Guidance for Industry, U.S. Department of Health and Human Services, Food and Drug Administration, Center for Drug Evaluation and Research (CDER), U.S.
Government Printing Office: Washington, DC: 1997. DOI: 10.1201/9780824741969.axb.

3. Diven, D. G.; Bartenstein, D. W.; Carroll, D. R. Extending shelf life just makes sense. Mayo Clin. Proc. 2015, 90, 1471-1474. DOI: 10.1016/j.mayocp.2015.08.007.

4. Lyon, R. C.; Taylor, J. S.; Porter, D. A.; Prasanna, H. R.; Hussain, A. S. Stability profiles of drug products extended beyond labeled expiration dates. J. Pharm. Sci. 2006, 95, 1549-1560. DOI: 10.1002/jps.20636.

5. Expiration Dating Extension, U.S. Food and Drug Administration website.https://www.fda.gov/emergency-preparednessand-response/mcm-legal-regulatory-and-policy-framework/ expiration-dating-extension. Accessed January 15, 2020.

6. Stark G. F. J.; Tucker, I. G. A study of the stability of some commercial drug dosage forms beyond their expiration dates. Pharm. J. 1997, 258, 637-640.

7. Yang, H. S.; Edinboro, L. Therapeutic drug monitoring of lacosamide by LC-MS/MS. Methods Mol. Biol. 2019, 1872, 6773. DOI: 10.1007/978-1-4939-8823-5_7.

8. Hurley, S. C. Lamotrigine update and its use in mood disorders. 
Ann. Pharmacother. 2002, 36, 860-873. DOI: 10.1345/ aph.1A102.

9. Shah, H. J.; Subbaiah, G.; Patel, D. M.; Patel, C. N. In vitro-in vivo correlation of modified release dosage form of lamotrigine. Biopharm. Drug Dispos. 2009, 30, 524-531. DOI: 10.1002/ bdd.688.

10. Stillhart, C.; Parrott, N. J.; Lindenberg, M.; Chalus, P.; Bentley, D.; Szepes, A. Characterising Drug release from immediate-release formulations of a poorly soluble compound, basmisanil, through absorption modelling and dissolution testing. AAPS J. 2017, 19, 827-836. DOI: 10.1208/s12248-017-0060-1.

11. Wagner, C.; Jantratid, E.; Kesisoglou, F.; Vertzoni, M.; Reppas, C.; J, B. D. Predicting the oral absorption of a poorly soluble, poorly permeable weak base using biorelevant dissolution and transfer model tests coupled with a physiologically based pharmacokinetic model. Eur. J. Pharm. Biopharm. 2012, 82, 127-138. DOI: 10.1016/j.ejpb.2012.05.008.

12. Newman, A.; Reutzel-Edens, S. M.; Zografi, G. Coamorphous active pharmaceutical ingredient-small molecule mixtures: considerations in the choice of coformers for enhancing dissolution and oral bioavailability. J. Pharm. Sci. 2018, 107, 5-17. DOI: 10.1016/j.xphs.2017.09.024.

13. Horter, D.; Dressman, J. B. Influence of physicochemical properties on dissolution of drugs in the gastrointestinal tract. Adv. Drug Deliv. Rev. 2001, 46, 75-87.

14. Pouton, C. W. Formulation of poorly water-soluble drugs for oral administration: physicochemical and physiological issues and the lipid formulation classification system. Eur. J. Pharm. Sci. 2006, 29, 278-287. DOI: 10.1016/j.ejps.2006.04.016.

15. Bhattachar, S. N.; Perkins, E. J.; Tan, J. S.; Burns, L. J. Effect of gastric $\mathrm{pH}$ on the pharmacokinetics of a BCS class II compound in dogs: utilization of an artificial stomach and duodenum dissolution model and GastroPlus, simulations to predict absorption. J. Pharm. Sci. 2011, 100, 4756-4765. DOI: 10.1002/jps.22669.

16. Klein, $\mathrm{S}$. The use of biorelevant dissolution media to forecast the in vivo performance of a drug. AAPS J. 2010, 12, 397-406. DOI: 10.1208/s12248-010-9203-3.

17. Emami, J.; Ghassami, N.; Ahmadi, F. Development and validation of a new HPLC method for determination of lamotrigine and related compounds in tablet formulations. J. Pharm. Biomed. Anal. 2006, 40, 999-1005. DOI: 10.1016/j.jpba.2005.07.045.

18. European Pharmacopoeia, 9th ed.; European Directorate for the Quality of Medicines \& Healthcare, Council of Europe: Strasbourg, France: 2016.

19. Podczeck, F. Methods for the practical determination of the mechanical strength of tablets--from empiricism to science. Int. J. Pharm. 2012, 436, 214-232. DOI: 10.1016/j. ijpharm.2012.06.059.

20. The United States Pharmacopeia and National Formulary. USP 41-NF 36; The United States Pharmacopeial Convention, Inc.:Rockville, MD, 2018. DOI: 10.4135/9781412963855.n1200.

21. Gibaldi, M.; Feldman, S. Establishment of sink conditions in dissolution rate determinations. Theoretical considerations and application to nondisintegrating dosage forms. J. Pharm. Sci. 1967, 56, 1238-1242. DOI: 10.1002/jps.2600561005.

22. Israel, G., Freguglia, P. Modelli matematici nelle scienze biologiche. 1998.

23. Higuchi, T. Mechanism of sustained-action medication. Theoretical analysis of rate of release of solid drugs dispersed in solid matrices. J. Pharm. Sci. 1963, 52, 1145-1149. DOI: 10.1002/ jps.2600521210.

24. Hixson, A.; Crowell, J. Dependence of reaction velocity upon surface and agitation. Industrial \& Engineering Chemistry. 1931, 23, 923-931. DOI: 10.1021/ie50260a018.

25. Costa, P.; Lobo, J. M. S. Modeling and comparison of dissolution profiles. Eur. J. Pharm. Sci. 2001, 13, 123-133.

26. Berry, M. R.; Likar, M. D. Statistical assessment of dissolution and drug release profile similarity using a model-dependent approach. J. Pharm. Biomed. Anal. 2007, 45, 194-200. DOI: 10.1016/j.jpba.2007.05.021.

27. Zhang, Y., Huo, M., Zhou, J., Zou, A., Li, W., Yao, C., Xie, S. DDSolver: an add-in program for modeling and comparison of drug dissolution profiles. AAPS J. 2010, 12(3), 263-271. DOI: 10.1208/s12248-010-9185-1.

28. Onur, E. Drug-excipient interaction in solid pharmaceutical dosage forms. Turk. Hij. Deney Biyol. Derg. 1976, 36, 88-104.

29. Fung, M. H.; DeVault, M.; Kuwata, K. T.; Suryanarayanan, R. Drug-excipient interactions: effect on molecular mobility and physical stability of ketoconazole-organic acid coamorphous systems. Mol. Pharm. 2018, 15, 1052-1061. DOI: 10.1021/acs. molpharmaceut.7b00932.

30. Wang, J.; Wen, H.; Desai, D. Lubrication in tablet formulations. Eur. J. Pharm. Biopharm. 2010, 75, 1-15. DOI: 10.1016/j. ejpb.2010.01.007.

31. Ariyasu, A.; Hattori, Y.; Otsuka, M. Delay effect of magnesium stearate on tablet dissolution in acidic medium. Int. J. Pharm. 2016, 511, 757-764. DOI: 10.1016/j.ijpharm.2016.07.034.

32. Desai, P. M.; Liew, C. V.; Heng, P. W. S. Review of disintegrants and the disintegration phenomena. J. Pharm. Sci. 2016, 105, 2545-2555. DOI: 10.1016/j.xphs.2015.12.019.

33. Li, X.; Jiang, C.; Pan, L.; Zhang, H.; Hu, L.; Li, T.; Yang, X. Effects of preparing techniques and aging on dissolution behavior of the solid dispersions of NF/Soluplus/Kollidon SR: identification and classification by a combined analysis by FT-IR spectroscopy and computational approaches. Drug Dev. Ind. Pharm. 2015, 41, 2-14. DOI: 10.3109/03639045.2014.938080.

34. Babu, R. J.; Pandit, J. K. Effect of aging on the dissolution stability of glibenclamide/beta-cyclodextrin complex. Drug Dev. Ind. Pharm. 1999, 25, 1215-1219. DOI: 10.1081/DDC-100102291.

35. Dash, S.; Murthy, P. N.; Nath, L.; Chowdhury, P. Kinetic modeling on drug release from controlled drug delivery systems. Acta Pol. Pharm. 2010, 67, 217-223.

36. Lokhandwala, H.; Deshpande, A.; Deshpande, S. Kinetic modeling and dissolution profiles comparison: an overview. Int. J. Pharm. Bio. Sci 2013, 4, 728-773. 